\title{
Slow and fast light effects in semiconductor waveguides for applications in microwave photonics
}

Mørk, Jesper; Öhman, Filip; Xue, Weiqi; Chen, Yaohui; Blaaberg, Søren; Sales, Salvador

Published in:

2008 International Topical Meeting on Microwave Photonics

Link to article, DOI:

10.1109/MWP.2008.4666699

Publication date:

2008

Document Version

Publisher's PDF, also known as Version of record

Link back to DTU Orbit

Citation (APA):

Mørk, J., Öhman, F., Xue, W., Chen, Y., Blaaberg, S., \& Sales, S. (2008). Slow and fast light effects in semiconductor waveguides for applications in microwave photonics. In 2008 International Topical Meeting on Microwave Photonics (pp. 210-213). IEEE. https://doi.org/10.1109/MWP.2008.4666699

\section{General rights}

Copyright and moral rights for the publications made accessible in the public portal are retained by the authors and/or other copyright owners and it is a condition of accessing publications that users recognise and abide by the legal requirements associated with these rights.

- Users may download and print one copy of any publication from the public portal for the purpose of private study or research.

- You may not further distribute the material or use it for any profit-making activity or commercial gain

- You may freely distribute the URL identifying the publication in the public portal 


\section{Slow and fast light effects in semiconductor waveguides for applications in microwave photonics}

\author{
Jesper Mørk, Filip Öhman, Weiqi Xue, Yaohui Chen, \\ Søren Blaaberg \\ DTU Fotonik, Department of Photonics Engineering, \\ Technical University of Denmark, Build. 343, \\ DK-2800 Lyngby, Denmark \\ jm@com.dtu.dk
}

We review the physics of slow and fast light effects in semiconductor waveguides. Different schemes for achieving optically or electronically controlled phase shifts are introduced and explained.

\section{INTRODUCTION}

The phenomenon of slow light has received a lot of attention since the spectacular experimental demonstration of light slow-down to cyclist speed [1]. Since then light has been brought to a complete stop and a number of different physical schemes and media have been explored. The interest in controlling the light of speed is motivated by gaining additional understanding of the fundamental nature of lightmatter interactions as well as the possibilities of exploiting these effects for various applications. In particular, the use of slow light effects for optical buffering has been suggested [2], but it has also been pointed out that there are basic physical limitations reflecting a compromise between the bandwidth that can be accommodated and the delay times that can be achieved [3]. On the other hand, the use of slow and fast light effects for controlling the phase of microwave signals imprinted as intensity modulation on an optical signal may have interesting applications within microwave photonics [4]. Two examples are the control of optically-fed phased array antennas as well as the synthesis and control of microwave filters. For practical applications it is of particular interest to use a medium enabling the realization of cheap and small devices and preferably also allowing integration with other functionalities. Semiconductor based devices are therefore of prime interest and a lot of work has been devoted to the exploration of slow and fast light effects in such structures. Unfortunately, the phenomenon of electromagnetically induced transparency, exploited so successfully in [1] is difficult to realize in semiconductor materials. Thus, dephasing times are very short and while a discrete level structure, necessary for implementing the scheme of electromagnetically induced transparency, can be realized using semiconductor quantum dots, the size fluctuations obtained using present-day technology lead to inhomogeneous

\author{
Salvador Sales \\ Institute of Telecomm. and Multimedia Applications, \\ Universidad Politécnica de Valencia, \\ Valencia, Spain
}

broadening that strongly diminishes the effect [2]. Instead, the use of an effect denoted coherent population oscillations (CPO), demonstrated in Ruby crystals [5], has been exploited by a number of different groups to achieve control of the light speed in semiconductor waveguides [6-15]. For recent reviews see $[8,10]$. In this paper we review the physical effect of CPO and outline several different schemes which may be used to enhance the phase shift and the frequency range.

\section{BASICS OF SLOW LIGHT}

A continuous wave $(\mathrm{CW})$ light beam propagating in a medium with refractive index $n$ has a phase velocity $v=c / n$, where $c$ is the velocity of light in vacuum. The refractive index is related to the relative dielectric constant $\varepsilon_{r}$ of the medium by $n^{2}=\varepsilon_{r}$. If the intensity of the signal varies in time, i.e. the spectrum of the signal has a finite width, the propagation speed of the intensity modulation is instead given by the group velocity

$$
v_{g}=\frac{c}{n_{g}}, \quad n_{g}=n+\omega \frac{d n}{d \omega}
$$

where $n_{g}$ is denoted the group refractive index and $\omega$ is the optical frequency. The group velocity thus differs from the phase velocity in media and at frequencies, where the refractive index has a non-zero first-order derivative with respect to frequency. If the light intensity is modulated, e.g. sinusoidally, the group velocity thus describes the velocity with which this intensity pattern is transmitted through the device. When discussing light slow-down, it is the latter term of Eq. (1), originating from dispersion of the medium that is of interest, since this part of the group index may potentially be changed, thus allowing controlling the speed of light. 


\section{COHERENT POPULATION OSCILLATIONS}

The CPO effect relies on the beating between external laser beams that excite the semiconductor, which leads to a modulation of the carrier distribution in the semiconductor and subsequent changes of the refractive index and its dispersion. The effect can in general be analyzed in the frequency domain using four-wave mixing (FWM) theory. However, in the practically important case where the external signal is generated by intensity modulating a laser beam, the refractive index dynamics can be neglected and the effect can be interpreted in the time domain as an effect of saturation [9]. In the case of an absorbing waveguide, i.e., an electroabsorber (EA), CPO effects lead to slow light, corresponding to a phase delay, while an amplifying waveguide, i.e. a semiconductor optical amplifier (SOA) leads to fast light, corresponding to a phase advance. In both cases the saturation power and the effective carrier lifetime are important characteristics governing the power and frequency dependence. Depending on the frequency difference between the laser beams, or the modulation frequency, different effects in the dynamics of the carrier distribution will be important. The structure of the active layer, i.e., bulk or lowdimensional, therefore influences the characteristics of the slow and fast light behaviour.

\section{CASCADING DEVICES}

Since EA structures display much shorter lifetimes than SOAs due to carrier sweep-out by the applied field, they are preferable for high-frequency applications, but on the other hand the absorption limits the transmitted intensity. A solution may be to combine the two structures - due to the different responses one can benefit from the slow light effect of the EA section while obtaining gain from the SOA section without the counteracting effect of light speed/up in that section [11]. Furthermore, by concatenating several such sections the total microwave phase delay can be increased [12]. Fig. 1 shows a photo and a schematic of a fabricated multi-section device and the corresponding measured phase change, in a contour diagram, as a function of input optical intensity and reverse bias. The figure shows that it is possible to control the degree of light slow-down either electrically through the reverse bias or optically through the intensity of the optical input signal. For fixed reverse bias we observe an optimum intensity, which is due to the saturation induced by the input signal [5] and for fixed optical input intensity an optimum reverse bias is observed, which reflects that the sweep-out time of carriers from the active region decreases for increasing voltage [9]. In this particular case a maximum phase change of about 140 degrees is obtained for fixed SOA currents and reverse voltages on the EA sections. If the electrical bias is allowed to change from forward to reverse bias, the absolute phase change could be further increased, as demonstrated recently in a single section waveguide [13].
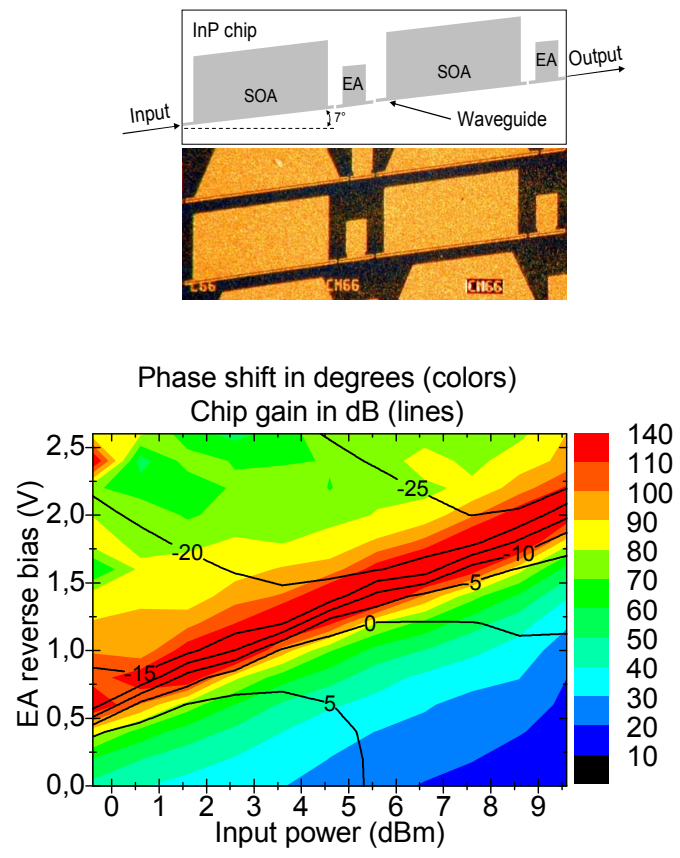

Figure 1. Schematic and photo of multi-electrode quantum well waveguide (upper) and contour plot of measured phase shift (lower). The measurements are carried out at a frequency of $4 \mathrm{GHz}$ and the contour diagram shows the phase shift (colour code) versus optical input power and reverse bias. Additional contour lines are shown for different levels of net waveguide transmission

\section{OPTICAL FILTERING}

It has been shown that for an intensity modulated, doublesideband, input signal the phase shift only depends on the gain dynamics $[9,10]$. However, the modulation of the refractive index, mediated by a non-zero linewidth enhancement factor, can be exploited to increase the phase shift by performing optical filtering prior to detection [14]. The experimental set-up is shown in Fig 1. The laser, with the wavelength of $1539.46 \mathrm{~nm}$, is modulated by the network analyzer through a push-pull Mach-Zehnder intensity modulator (MZM) with a residual chirp $\alpha$ res $=-0.2$, to generate two sidebands (red-shifted and blue-shifted) in addition to the strong carrier. After passing through a bulk SOA, where FWM effects will induce changes of the phase and the amplitude of the two sidebands, one of the two sidebands, red-shifted or blue-shifted, is blocked by a FBG notch filter with $0.1 \mathrm{~nm}$ bandwidth before detection. When the modulation frequency is larger than $4 \mathrm{GHz}$, one sideband can easily be removed without disturbing the other sideband or the carrier. By incorporating an optical fiber amplifier (EDFA) and a variable optical attenuator (VOA), the input optical power can be adjusted between $-10.3 \mathrm{dBm}$ and $13.6 \mathrm{dBm}$. 

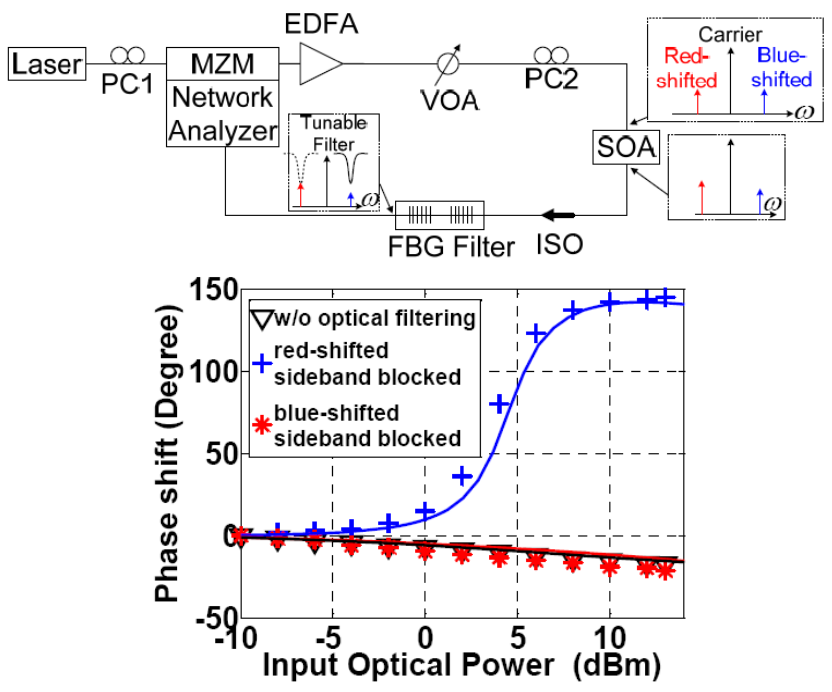

Figure 2. Experimental set-up and results for the case of performing optical filtering before detection. A fiber-grating notch filter allows to block one of the sidebands before detection. The modulation frequency is $19 \mathrm{GHz}$. Markers are experimental data and solid lines are numerical simulations.

The experimental results are shown as markers in Fig. 2 for three different cases, i.e., no filtering (black), blocking the blue sideband and letting the red pass (red) and blocking the red sideband and letting the blue pass (blue). The results are also compared with numerical simulations (solid lines) based on a wave mixing model, showing very good agreement. The results show that the absolute phase shift as well as the frequency of operation can be significantly enhanced by blocking the red-shifted sideband. On the other hand the blocking of the blue-shifted sideband only leads to a small change in the phase shift. This behaviour can be explained by considering the effective gain and phase change induced on the two sidebands via wave mixing, for which the phase relations beteeen the different components play an important role [15].

\section{EXPLOITING POLARIZATION ROTATION}

Finally, we demonstrate a quite different method for realizing controllable microwave phase shifts by exploiting polarization effects. The experimental set-up is shown in Fig. 3. The laser beam, with the wavelength of $1550 \mathrm{~nm}$, is sinusoidally modulated by the network analyzer through a MZM which is biased to make sure that the TE and TM components are operated at slopes of the transfer curve of opposite sign. In this way a $180^{\circ} \mathrm{RF}$ phase difference $\phi$ between the modulated TE and TM components is achieved. By exploiting intensity dependent polarization rotation in an SOA and introducing a polarization selective component before photodetection, the phase shift can thus be controlled. In the experiment, by incorporating an EDFA and Variable Optical Attenuator (VOA), the input optical power to the SOA is tuneable between $-7 \mathrm{dBm}$ and $13 \mathrm{dBm}$, which will induce polarization rotation of the signal in the SOA [14]. After the SOA, a polarization controller (PC3) and polarizati-
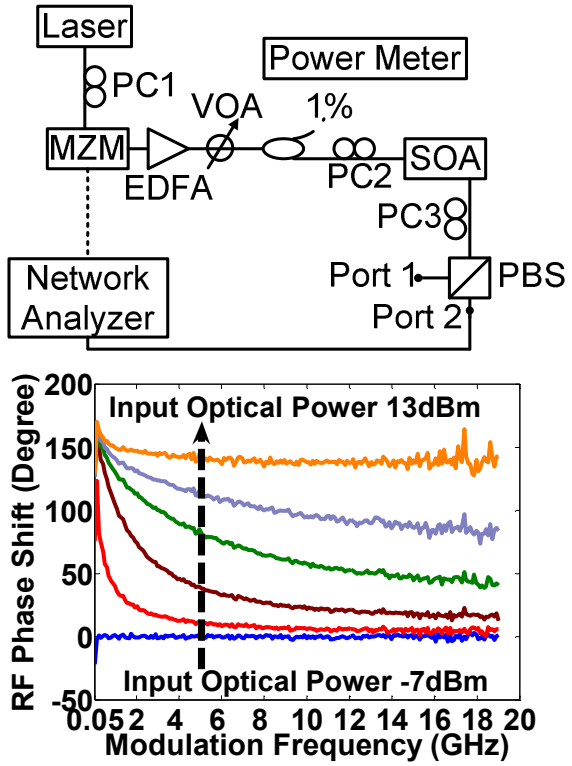

Figure 3. (Upper) Experimental set-up for exploiting polarization rotation in an SOA to accomplish control of the microwave phase. (Lower) Measured microwave phase shift versus modulation frequency for different values of input optical power ranging from $-7 \mathrm{dBm}$ (lower trace) to $13 \mathrm{dBm}$ (upper trace)

on beam splitter (PBS) are used to select the polarization to be detected by the network analyzer. The pump current of the SOA is fixed at $160 \mathrm{~mA}$. The RF modulation power is $0 \mathrm{dBm}$. The measurement results show that the phase can be continuously tuned via the input optical power and a controllable phase shift of about $150^{\circ}$ is obtained at a modulation frequency as high as $19 \mathrm{GHz}$.

\section{CONCLUSIONS}

We have introduced and demonstrated different schemes for controlling the phase shift of an intensity modulated optical signal, based on slow and fast light effects in semiconductor optical waveguides. We find that the basic effect of wave mixing in the active region can be further enhanced by cacscading devices or exploiting optical filtering. Also, phase shifters may be realized by introducing different phase shifts onto the two polarization components of the optical field and continuously switching between these by using the effect of nonlinear polarization rotation. At the moment maximum phase shifts achieved are on the order of $180^{\circ}$ and are obtained at frequencies up to about $20 \mathrm{GHz}$. In order to implement phased array antennas and microwave filters with sufficient flexibility the controllable phase shift should be further pushed to 360 degrees and additional requirements may apply depending on the application..

\section{ACKNOWLEDGMENT}

The authors acknowledge support from the Danish Research Councils through the project QUEST as well as the EU through the $7^{\text {th }}$ framework progamme GOSPEL. 


\section{REFERENCES}

[1] L. V. Hau, S. E. Harris, Z. Dutton and C. H. Behroozi, "Light speed reduction to 17 meters per second in an ultracold atomic gas." Nature vol. 397, pp. $594-598,1999$.

[2] C. Chang-Hasnain, P.-C. Ku, J. Kim and S.-L. Chuang, "Variable optical buffer using slow-light in semiconductor nanostructures." Proc. IEEE, vol. 91, pp. 1884, 2003.

[3] R. S. Tucker, P.-C. Ku and C. J. Chang-Hasnain, "Slow-light optical buffers: capabilities and fundamental limitations." J. Lightwave Technol. vol. 23, pp. 4046-4066, 2005.

[4] J. Capmany et al., "Discrete-time optical processing of microwave signals", J. Lightwave Technol., vol. 23, pp. 702, 2005.

[5] M. S. Bigelow, N. N. Lepeshkin and R. W. Boyd, "Observation of ultraslow light propagation in a ruby crystal at room temperature." Phys. Rev. Lett., vol. 90, pp. 113903-1-4, 2003.

[6] P. Ku, F. Sedgwick, C. J. Chang-Hasnain, P. Palinginis, T. Li, H. Wang, S. Chang and S. Chuang, "Slow light in semiconductor quantum wells." Opt. Lett., vol. 29, pp. 2291, 2004.

[7] P. Palinginis, S. Crankshaw and F. Sedgwick, "Ultra-slow light $(<200$ $\mathrm{m} / \mathrm{s}$ ) propagation in a semiconductor nanostructure. Appl. Phys. Lett., vol. 87, pp. 171102, 2005.

[8] C Chang-Hasnain and S. L. Chuang, "Slow and Fast Light in Semiconductor Quantum-Well and Quantum-Dot Devices", J. Lightwave Technol., vol. 24, pp. 4642, 2006.
[9] J. Mørk, R. Kjær, M. van der Poel and K. Yvind, "Slow light in a semiconductor waveguide at gigahertz frequencies." Opt. Express, vol. 13, pp. 8136-8145, 2005.

[10] J. Mørk, F. Öhman, M. van der Poel, Y. Chen, P. Lunnemann and K. Yvind, "Slow and fast light: Controlling the speed of light using semiconductor waveguides", Laser and Photonics Reviews, to appear, 2008.

[11] F. Öhman, K. Yvind and J. Mørk, "Slow Light in a Semiconductor Waveguide for True-Time Delay Applications in Microwave Photonics”, IEEE Photon. Technol. Lett., vol. 19, 1145-1147, 2007.

[12] F. Öhman, K. Yvind, and J. Mørk, "Voltage-controlled slow light in an integrated semiconductor structure with net gain", Optics Express, Vol. 14, 9955-9962, 2006.

[13] P. Kondratko, et al., "Slow-to-fast light using absorption to gain switching in quantum-well semiconductor optical amplifier," Opt. Express, vol. 15, pp. 9963, 2007.

[14] W. Xue, Y. Chen, F. Öhman, S. Sales, and J. Mørk, "Enhancing Light Slow-Down in Semiconductor Optical Amplifiers by Optical Filtering", Opt. Lett., vol. 33, 1084-1086, 2008.

[15] Y. Chen, W. Xue, F. Öhman, and J. Mørk, "Theory of OpticalFiltering Enhanced Slow and Fast Light Effects in Semiconductor Optical Waveguides, " submitted for publication in IEEE J. Lightwave Technol., 2008 